\title{
TAHSP:-
}

The Internet Joưnal of Allied Health Sciences and Practice

A Peer Reviewed Publication of the College of Health Care Sciences at Nova Southeastern University

Dedicated to allied health professional practice and education

http://ijahsp.nova.edu Vol. 12 No. 1 ISSN 1540-580X

\section{The Correlation Between Sleep Efficiency and the Risk of Obstructive Sleep Apnea}

\author{
Mazen Munaji Homoud, BS, MSIS, RRT-SDS1
}

1. Graduate Student, Interdisciplinary Studies Program, Texas State University, San Marcos, Texas

United States

CITATION: Homoud M. The Correlation Between Sleep Efficiency and the Risk of Obstructive Sleep Apnea. The Internet Journal of Allied Health Sciences and Practice. Jan 2014. Volume 12 Number 1.

\begin{abstract}
Purpose: The purpose of this correlation research was to identify the relationship between Obstructive Sleep Apnea (OSA) and sleep efficiency. Subjects: A total of six men and twelve women $(\mathrm{N}=18)$ form the College of Health Professions fulfilled the requirements for inclusion in this study. Method: This research integrated two investigation methods. 1) STOP-BANG questionnaire was completed by students and faculty from the College of Health Professions at Texas State University. Then, 2) each participant wore an Actigraphy watch for seven days. The result of the STOP-BANG questionnaire was compared to the result of the Actigraphy in regard to low or high risk of OSA and the normal or below normal sleep efficiency. Results: There was no significant relationship between the risk of having OSA according to STOP-BANG and sleep efficiency measured by Actigraphy watch in undiagnosed individuals $(r=.240, p=.337)$. However, there was a positive, significant relationship between age and sleep efficiency. Conclusion and Recommendations: In order to improve the external validity, more participants need to be included in future research. Moreover, the use of medication that could affect sleep and alcohol consumption need to addressed. A full night sleep study in a sleep lab adds a standard for comparison. Finally, using other questionnaires or advanced forms of Actigraphy technology could provide better results.
\end{abstract}

\section{INTRODUCTION}

For the last three decades, sleep and sleep medicine have gained an increase in attention, given that people spend around one third of their lifespan sleeping. ${ }^{1}$ Moreover, healthcare practitioners have found a strong association between sleep disorders and serious health conditions that affect an individual's life and society in general. ${ }^{2}$

Obstructive Sleep Apnea (OSA) is a common sleep disorder and is estimated to have a prevalence of $9 \%$ in women and $24 \%$ in men. ${ }^{3}$ There is a belief that approximately $93 \%$ of women and $82 \%$ of men with moderate to severe OSA remain undiagnosed. ${ }^{4}$ Since undiagnosed OSA is a serious issue that costs the United States around 4.3 billion dollars per year in medical costs alone, patients with OSA are at greater risk for having serious conditions, such as cardiovascular morbidities, if left untreated. ${ }^{5}$ In addition, OSA has a negative impact on productivity and quality of life because it increases day time sleepiness and fatigue, memory loss, and reduced libido.

In 2010, Kapur defined OSA as a disorder characterized by recurring collapsing and opening of the upper airway during sleep. ${ }^{6}$ The disruption of sleep (arousal) is the result of an increase in the work of breathing as the body is trying to handle the abnormal low oxygen (hypoxemia) and high carbon dioxide (hypercapnia) in the blood. 6 Generally, people with OSA have greater instability of their ventilatory control during sleep when the respiratory system is under automatic control. There are numerous factors that contribute to the pathogenesis of OSA such as upper-airway anatomy and its dilator muscle activity during sleep, genetics, gender, obesity, lung volume, ventilatory control stability, age, sleep state stability, and rostral fluid shifts. Therefore, individuals with several of these factors are more susceptible to having OSA that leads to changes in sleep efficiency. 
Sleep efficiency is a quantitative sleep parameter that is calculated by dividing the time of sleep in minutes by the time in bed in minutes. Normal sleep efficiency is approximately 85 to $90 \%$. A meta-analysis of 65 studies published between 1960 and 2003 revealed a strong negative correlation between sleep efficiency and age in healthy individuals. It is the only parameter that continues to significantly decrease after the age of $60 . .^{7}$ It is an important sleep parameter that is used frequently to support new diagnosis methods. For example, sleep efficiency has been used to support the use of unattended home-based polysomnography. ${ }^{8}$

Several studies have suggested that early diagnosis of OSA would improve patients' outcomes and quality of life. Therefore, health practitioners around the world are trying to reduce this gap between diagnosed and undiagnosed OSA patients by using the best method. Polysomnography is still considered the gold standard for diagnosing OSA. However, the test has some disadvantages such as high cost, the need for skillful technicians, and the number of available laboratories that can accommodate the increasing suspected OSA population.5,9,10 Therefore, other options that are cheaper and can be used as an initial assessment were explored. One of the suggested tools was a questionnaire to screen patients before they go for polysomnography.

In 2008, the STOP-BANG questionnaire was published. It contains eight "yes" or "no" questions, and its framework was based on the Berlin questionnaire that was released on 1999. The Berlin questionnaire consisted of 10 questions that were directly related to the signs and symptoms of the disease. ${ }^{11}$ For identifying moderate or severe OSA, the Berlin and the STOP-BANG questionnaires had the highest sensitivity and specificity. ${ }^{11-13} \mathrm{Abrishami}$ et al ${ }^{9}$ states that questionnaires provide the appropriate tools and can be part of daily practice for quick prediction of OSA. Newer questionnaires, such as STOP and STOP-Bang, have fewer and more straightforward questions and achieve higher response rates. ${ }^{9}$

However, questionnaires are not the recommended tool. The technology of Actigraphy could be used in order to lower the cost of identified undiagnosed OSA. Elbaz et al used the technology of Actigraphy, which records the intensity of movement over time. These researchers removed the neurophysiologic variables and included an Actigraphy watch instead. The result indicated that using simplified polygraphy with an Actigraphy watch may assist in the diagnosis of OSA. ${ }^{14}$ Furthermore, the watch demonstrated reasonable validity and reliability in detecting wakefulness within sleep periods for healthy individuals. ${ }^{15}$ Another study revealed a strong positive correlation of 0.89 between polysomnography and Actigraphy for total sleep time and 0.71 for sleep efficiency ${ }^{16}$

The sleep period in polysomnography (gold standard) is detected by EEG and EMG activities. Every epoch of "awake" after the first onset of sleep is added together for a total arousal time. The Actiware software (for the Activity watch) translates the row data according to the level of activity; the "sleep period" is when there is no or minimal activity.

\section{METHODS \\ Design}

The purpose of the correlation research study was to identify the relationship between Obstructive Sleep Apnea (OSA) and sleep efficiency. If the result indicated a strong relationship, then this method could be used to reduce health costs of both untreated undiagnosed OSA and unnecessary PSG.

The research question for this current study integrated OSA and sleep efficiency: R1: What is the relationship, if any, between the risk of having OSA predated by the STOP-BANG questionnaire and sleep efficiency measured by Actigraphy watch?

In consideration of using two different measurement tools and one research question, the study included the following hypotheses:

$\mathrm{H}_{0}$ : There is no correlation between the risk of having OSA measured by the STOP-BANG questionnaire and sleep efficiency measured by the Actigraphy watch.

$H_{1}$ : A correlation exists between the risk of having OSA measured by the STOP-BANG questionnaire and sleep efficiency measured by the Actigraphy watch.

\section{Participants}

Participants were recruited through emails and word of mouth. An email was designed by the research team and then forwarded to other department heads through the Texas State University email system, who in turn contacted their students accordingly. Participants contacted the research team electronically though the university's email to set face-to-face appointment with the researcher. During the scheduled appointment, the researcher explained the purpose of the study, which was the use of the Actigraphy watch. Then, if the participant agreed, he or she would sign a consent form, fill out a STOP-BANG questionnaire, and 
finally wear an Actigraphy watch for one week. Moreover, there were no specific exclusion criteria for participation in this study other than English language illiteracy. Participants were also asked to indicate their age, race, department, and classification as faculty or student.

\section{Tools}

Dr. F. Chung, based on the Berlin questionnaire that was developed in 1999 Berlin, Germany, developed the STOP-BANG questionnaire in 2008. It consists of eight self-reporting "yes" or "no" questions. Each of these questions represent signs and symptoms that are presented in OSA patients as well as abbreviation letters. S for Snoring; T for Tiredness; $\mathrm{O}$ for Obstruction; $\mathrm{P}$ for High blood pressure; B for Body Mass Index $>35 \mathrm{~kg} / \mathrm{m2}$; A for Age $>50$; $\mathrm{N}$ for Neck circumflex Male $>17^{\prime \prime}$, and Female $>$ 16"; and G for Gender Male > Female. In order to be qualified as a high risk for OSA, participants must check at least 3 'yes' on the STOP- BANG questionnaire. The more "yes's," the higher the risk. The sensitivity of the questionnaire increases from $78.4 \%$ if a subject answered "yes" to the first four questions to $100 \%$ if a subject answered "yes" to all eight questions. "11 Table 1 shows the STOP-BANG questionnaire.

WN

Table 1. STOP-BANG Sleep Apnea Questionnaire

Faculty / Student

Age

Please indicate your Major

\begin{tabular}{|c|c|c|}
\hline STOP-BANG & YES & NO \\
\hline \multicolumn{3}{|c|}{ Do you SNORE loudly (louder than talking or loud enough to be heard through closed doors)? } \\
\hline \multicolumn{3}{|c|}{ Do you often feel TIRED, fatigued, or sleepy during daytime? } \\
\hline \multicolumn{3}{|l|}{ Has anyone OBSERVED you stop breathing during your sleep? } \\
\hline \multicolumn{3}{|l|}{ Do you have or are you being treated for high blood PRESSURE } \\
\hline \multicolumn{3}{|l|}{$\begin{array}{l}\text { Do you have BMl more than } 35 \mathrm{~kg} / \mathrm{m} 2 ? \\
\text { If you do not know, see the table below }\end{array}$} \\
\hline \multicolumn{3}{|l|}{ Is your AGE over 50 years old? } \\
\hline \multicolumn{3}{|l|}{ Do you have a NECK circumference $>15.75$ inches $(40 \mathrm{~cm})$ ? } \\
\hline Are you Male GENDER? & & \\
\hline
\end{tabular}

\section{Actigraphy}

This study utilized the Mini-Mitter Actiwatch-2 (PRMM) from Respironics that records body movement using a piezoelectric accelerometer and a light sensor to provide three data channels: photopic white light illuminance, activity, and event marking. It stores up to 15 days' worth of data in its $1 \mathrm{MB}$ internal memory. The watch has a rechargeable battery that can last up to 30 days. Participants were instructed to wear the device on the non-dominant wrist for seven days. During this period, data were collected in 1-min epochs. The collected data were analyzed through Actiware software version 5.0 with the default setting that uses a medium sensitivity threshold of (40 counts per epoch).

The Actiware software provides graphs where the $x$-axis shows the time and the $y$-axis shows the activity level. These graphs give a good general description of the patient's activity level throughout the day. During sleep, participants demonstrate a very low or no activity. Any activity after initiation of sleep will be counted as arousal and will be added together for a total Wake After Sleep Onset (WASO) time in minutes. ${ }^{17}$ It provides a report that contains total sleep time (TST), wake after sleep onset (WASO), and sleep efficiency (SE) in addition to other outcomes that were not included in this research. ${ }^{18}$ Twelve Actigraphy watches and a computer with the Actiware software were provided for free from the Respiratory Care Department at Texas State University.

\section{Statistically Analyses}

The data were transferred from nonnumeric to numeric in an excel spreadsheet. The data were then imported to SPSS software version 21 for analysis. The Pearson correlation was utilized assuming that the two variables were measured on at least interval scales. The Pearson correlation determined the extent to which values of the two variables are proportional to each other. ${ }^{19}$

\section{RESULTS}

A total of six men and twelve women ( $\mathrm{N}=18)$ from the College of Health Professions completed the questionnaire (table 2). The participants were between the ages of 21 and 65 at the time of the research. Twenty-eight percent were college faculty and $72 \%$ were students. Thirty-nine percent of the participants were from the nursing department, $28 \%$ from respiratory care department, $22 \%$ from physical therapy department, and $11 \%$ from communication disorders department. 
Table 2. Participant Data

\begin{tabular}{|l|l|l|l|}
\hline Demographic Variables & Frequency & Percent \\
\hline Gender & Men & 6 & 33.3 \\
& Women & 12 & 66.7 \\
& Total & 18 & 100 \\
\hline Age & Minimum & & \\
& Maximum & 21 & \\
& Mean & 65 & \\
& Range & 35.2 & \\
& Standard Deviation & 40 & \\
\hline Classification & 13.6 & 27.8 \\
& Faculty & 5 & 72.2 \\
\hline Departments & Student & 13 & 38.9 \\
& & & 27.8 \\
& Nursing & 7 & 22.2 \\
& Respiratory Care & 5 & 11.1 \\
& Physical Therapy & 4 & \\
\hline
\end{tabular}

The findings indicated that the null hypothesis was not rejected in this study. There was no significant relation between the risk of having OSA according to STOP-BANG and sleep efficiency measured by an Actigraphy watch in undiagnosed individuals $(r$ $=.240, p=.337)$.

According to STOP-BANG questionnaire results, five of the participants were at high risk of having OSA. Only 4 of them were found to have lower sleep efficiency. On the other hand, the Actigraphy result indicated a total 11 participants had low sleep efficiency.

\begin{tabular}{|l|l|l|l|}
\hline \multicolumn{5}{|l|}{ Table 3. Results } \\
\hline Results & High risk & Frequency & Percent \\
\hline STOP-BANG & Low risk & 5 & 27.8 \\
& & 13 & 72.2 \\
& & 18 & 100 \\
\hline Sleep Efficiency & Normal & 7 & 38.9 \\
& Below normal & 11 & 61.1 \\
& & 18 & 100 \\
\hline
\end{tabular}

The only significant data were between sleep efficiency and age $(r=.471, p=.48)$, which is contradictory to the Ohayon et al meta-analysis study that indicated a significant continuous decline in sleep efficiency with age. ${ }^{7}$ 
Figure 1. Sleep Efficiency Data Collected from the Actigraphy Watch in Relation to Age

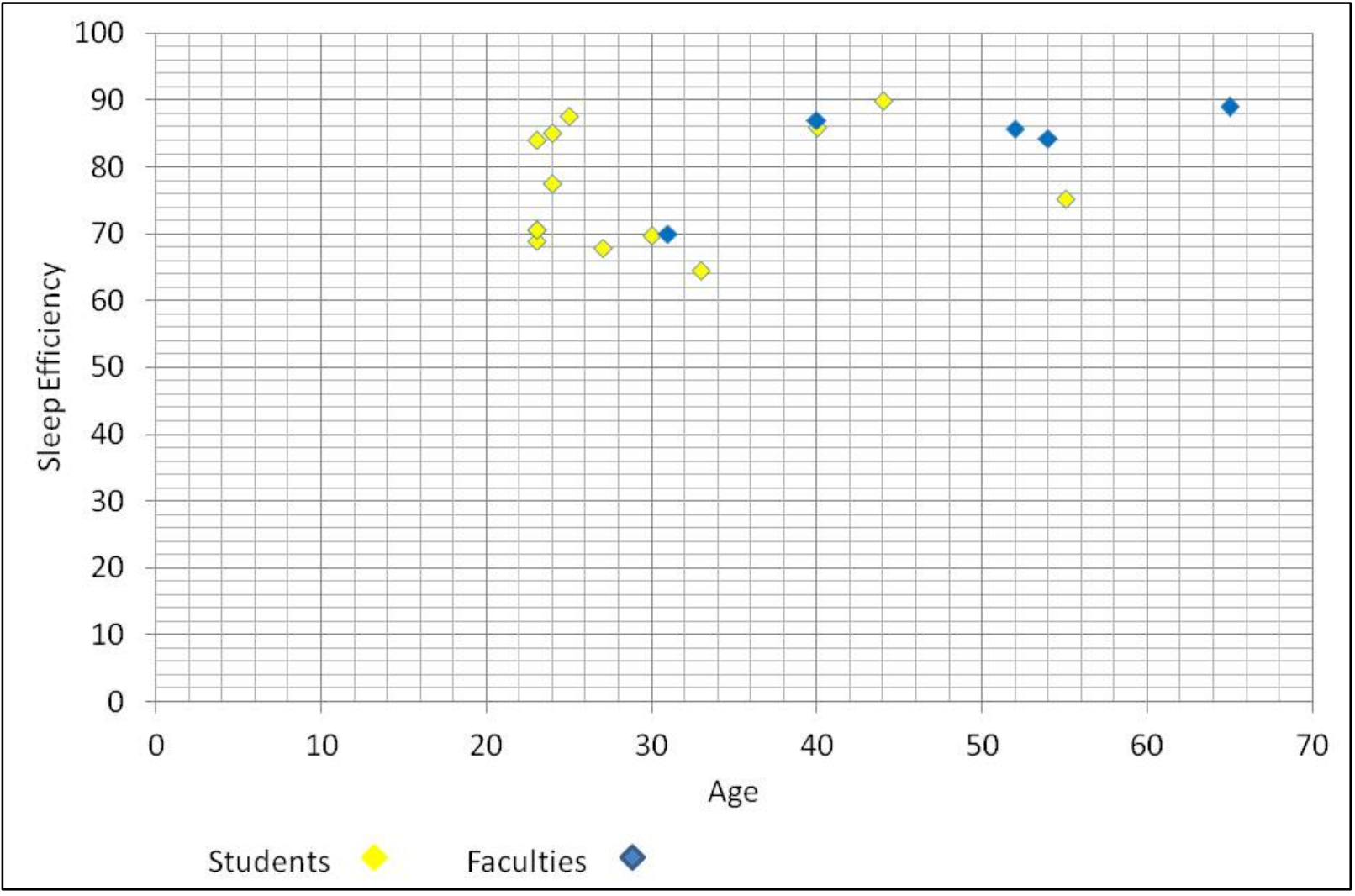

Descriptive Statistical Analysis

Table 4.Sleep Efficiency

\begin{tabular}{|l|l|}
\hline Sleep Efficiency & Percent \\
\hline Minimum & $64.67 \%$ \\
Maximum & $89.99 \%$ \\
Mean & $78.59 \%$ \\
Range & $25.32 \%$ \\
Standard Deviation & $8.7 \%$ \\
\hline
\end{tabular}

Table 5. Average Sleep Time

\begin{tabular}{|l|l|}
\hline Average Sleep Time & Minutes \\
\hline Minimum & 303 \\
Maximum & 478 \\
Mean & 366 \\
Range & 175 \\
Standard Deviation & 47.0 \\
\hline
\end{tabular}

Table 6. Average Number of Wakes

\begin{tabular}{|l|l|}
\hline Average Number of Wakes & Frequency \\
\hline Minimum & 13 \\
Maximum & 54 \\
Mean & 36 \\
Range & 41 \\
Standard Deviation & 9.2 \\
\hline
\end{tabular}


Table 7. Wake After Sleep Onset

\begin{tabular}{|l|l|}
\hline Wake After Sleep Onset & Minutes \\
\hline Minimum & 13.57 \\
Maximum & 160.99 \\
Mean & 73.79 \\
Range & 147.42 \\
Standard Deviation & 48.87 \\
\hline
\end{tabular}

Table 8.Sleep Efficiency Across Classification

\begin{tabular}{|l|l|l|}
\hline Sleep Efficiency & Faculty & Students \\
\hline Minimum & 69.94 & 64.67 \\
Maximum & 88.95 & 89.99 \\
Mean & 83.19 & 76.82 \\
\hline
\end{tabular}

\section{CONCLUSION}

Even though a significant correlation between the risk of OSA and sleep efficiency could not be establish using the tools in this study, there was a positive, significant relationship between age and sleep efficiency. However, this result was expected since $80 \%$ of the faculty subjects were over the age 35 .

\section{RECOMENDATIONS}

In order to improve the external validity, more participants need to be included in future research. Moreover, the use of medication that could affect sleep and alcohol consumption need to addressed. A full night sleep study in a sleep lab adds a standard for comparison. Finally, using other questionnaires or an advanced form of Actigraphy technology could provide better results

\section{LIMITATIONS}

Time limitation was a major factor in this study since the time allowed for collecting the data and presenting it was limited to four months with the exclusion of exam time because most students will have a disruptive sleep pattern during this period. Four watches broke down during the study; they either did not store any data or data could not be retrieved.

\section{REFERENCES}

1. Kumar VM. Sleep and sleep disorders. Indian J Chest Dis Allied Sci. 2008 Jan-Mar;50(1):129-35. [PMID 18610697]

2. Leger D, Bayon V, Laaban JP, Philip P. Impact of sleep apnea on economics. Sleep Med Rev. 2012 Oct;16(5):455-62. [PMID 22244357]

3. Frey WC, Pilcher J. Obstructive sleep-related breathing disorders in patients evaluated for bariatric surgery. Obes Surg. 2003;13(5):676-83. [PMID 14627460]

4. Young T, Evans L, Finn L, Palta M. Estimation of the clinically diagnosed proportion of sleep apnea syndrome in middleaged men and women. Sleep. 1997 Sep;20(9):705-6. [PMID 9406321]

5. Kapur V, Blough DK, Sandblom RE, Hert R, de Maine JB, Sullivan SD, Psaty BM. The medical cost of undiagnosed sleep apnea. Sleep. 1999;22(6):749-56. [PMID 10505820]

6. Kapur VK. Obstructive sleep apnea: diagnosis, epidemiology, and economics. Respir Care. 2010 Sep;55(9):1155-67. [PMID 20799998]

7. Ohayon MM, Carskadon MA, Guilleminault C, Vitiello MV. Meta-analysis of quantitative sleep parameters from childhood to old age in healthy individuals: developing normative sleep values across the human lifespan. Sleep. 2004 Nov 1;27(7):125573. [PMID 15586779]

8. Bruyneel M, Sanida C, Art G, Libert W, Cuvelier L, Paesmans M, Sergysels R, Ninane V. Sleep efficiency during sleep studies: results of a prospective study comparing home-based and in-hospital polysomnography. J Sleep Res. 2011 Mar;20(1 Pt 2):201-6. [PMID 20561176]

9. Abrishami A, Khajehdehi A, Chung F. A systematic review of screening questionnaires for obstructive sleep apnea. Can J Anaesth. 2010 May;57(5):423-38. [PMID 20143278]

10. Prasad B, Carley DW, Herdegen JJ. Continuous positive airway pressure device-based automated detection of obstructive sleep apnea compared to standard laboratory polysomnography. Sleep Breath. 2010 Jun;14(2):101-7. [PMID 19826848]

11. Chung F, Yegneswaran B, Liao P, Chung SA, Islam S, Khajehdehi A, Shapiro C. STOP Questionnaire: A tool to screen patients for obstructive sleep apnea. Anesthesiology. 2008;108(5):812-21. [PMID 18431116] 
12. Chung F, Yegneswaran B, Liao P, Chung SA, Vairavanathan, Islam S, Khajehdehi A, Shapiro C. Validation of the Berlin Questionnaire and American Society of Anesthesiologists checklist as screening tools for obstructive sleep apnea in surgical patients. Anesthesiology. 2008;108(5):822-30. [PMID 18431117]

13. Vana KD, Silva GE, Goldberg R. Predictive abilities of the STOP-Bang and Epworth Sleepiness Scale in identifying sleep clinic patients at high risk for obstructive sleep apnea. Res Nurs Health. 2013 Feb;36(1):84-94. [PMID 23007730]

14. Elbaz M, Roue GM, Lofaso F, Quera Salva MA. Utility of actigraphy in the diagnosis of obstructive sleep apnea. Sleep. 2002 Aug 1;25(5):527-31. [PMID 12150319]

15. Sadeh A. The role and validity of actigraphy in sleep medicine: an update. Sleep Med Rev. 2011 Aug;15(4):259-67. [PMID 21237680]

16. Dick R, Penzel T, Fietze I, Partinen M, Hein H, Schulz J. AASM standards of practice compliant validation of actigraphic sleep analysis from SOMNOwatch ${ }^{\mathrm{TM}}$ versus polysomnographic sleep diagnostics shows high conformity also among subjects with sleep disordered breathing. Physiol Meas. 2010 Dec;31(12):1623-33. [PMID 21071830]

17. Symanzik J, Shannon W. Exploratory graphic for functional actigraphy data. WNAR-JSM. 2008:3707-14.

18. Actigraphy. Philips Web site. http://www.healthcare.philips.com/main/homehealth/sleep/actiwatch/default.wpd. Accessed September 18, 2013.

19. Hill T, Lewicki P. Methods and applications. Statistics. 2006 Dec; 12(1):25-9. 